\title{
IMPURITY-INDUCED STRENGTHENING PHENOMENA IN ALKALI HALIDE CRYSTALS*
}

\author{
D. NowaK-Wożny, M. SuszyńșKa and M. Szmida
}

W. Trzebiatowski Institute of Low Temperature and Structure Resarch

Polish Academy of Sciences, pl. Katedralny 1, 50-950 Wroclaw, Poland

(Received April 19, 1991)

It has been shown that divalent impurities $\left(\mathrm{Sr}^{2+}, \mathrm{Eu}^{2+}\right)$ harden the $\mathrm{NaCl}$ monocrystals intensively. For the solution treated samples the effective obstacles and interaction mechanisms are nearly the same for both crystal systems. In contrary, because of some differences in the solubility limit and in the clustering phenomena as well, different interaction mechanisms determine the crystal strength attainable in as-received samples.

PACS, numbers: $77.40 .+\mathrm{i}$

\section{Introduction}

Plastic flow of crystalline materials is determined by the resistance to motion of fresh dislocations introduced by plastic deformation, and this resistance may arise in a number of ways. The understanding of strengthening mechanisms is usually based on the studies of the temperature and concentration dependence of the crystal strength increments induced by effective obstacles.

The aim of this paper is to present some experimental results concerning the hardening effects induced by divalent strontium and europium ions in $\mathrm{NaCl}$ monocrystals. The correlations between the state of aggregation and/or precipitation of these impurities and the mechanical response of the matrix could provide relevant data to check models of hardening processes originally developed for metallic systems. There are many advantages in using the alkali halides for the check of these models and the following can be exemplified. The crystallographic

*Part of this work has been presented at the Sixth Europhysics Topical Conference LATDIM-90, Groningen, Holland, 1990. 
and electronic structures of these systems are very simple and well-known. The host crystals are transparent in a wide spectral range enabling the identification of optically active centres. Different defects can be introduced in a controllable way, and different obstacles for the dislocation motion can easily be formed on the basis of the same point defects. At last, but not least, it should be stressed that the mechanical response of these crystal systems frequently resembles that typical of the $f c c$ metal alloys. For interpretation of the effects studied the following mechanisms were considered: Fleischer-[1], Snoek-[2] and Cottrel-type interactions [3] for the phenomena detected in ST-samples, and Kelly/Nicholson-cutting [4] as well as Orowan-looping [5] for AR specimens.

\section{Experimental details}

Room temperature (RT) yield stress $\left(\sigma_{0}\right)$ of $\mathrm{Sr}^{2+}$ - and $\mathrm{Eu}^{2+}$-doped $\mathrm{NaCl}$ monocrystals was measured as a function of the dopant concentration $\left(c_{2+}\right)$ for both as-received (AR) and solution treated (ST) samples. The effect of testing temperature $\left(T_{\text {test }}=192 \div 823 \mathrm{~K}\right)$ upon $\sigma_{0}$ was studied for ST-samples of $\mathrm{NaCl}: \mathrm{Sr}^{2+}$, whereas $\sigma_{0}$ of additionally annealed $\left(T_{\mathrm{an}}=\mathrm{RT}-523 \mathrm{~K}\right) \mathrm{ST}$-samples was measured for the $\mathrm{NaCl}: \mathrm{Eu}^{2+}$ crystals. Testing and thermal pretreatment procedures were the same as described elsewhere $[6,7]$.

The crystals were grown in our laboratory by a modified Bridgman method which enables to keep the content of oxygen-like contamination below the optically detectable limit. The concentration of the dopants was determined by the atomic absorption analysis.

\section{Results and discussion}

\subsection{Room-Temperature crystal strength}

\subsubsection{ST-samples}

In this case the elastic interactions between moving dislocations and the impurity-vacancy (I.V.) dipoles should determine the crystal strength. Two theories describe these interactions yielding $\sigma_{0} \propto\left(c_{2+}\right)^{n}$, with $n=0.5$ for rigid obstacles [1] and $n=1$ for dipole reorientation in the stress fields of moving dislocations [2]. However, a statistical theory of the solid solution hardening, which considers the distribution of interacting forces and distances between neighbouring obstacles along the dislocations, gives $n$ being equal to 0.66 [8].

ST-curves in Fig. 1 show that for small concentrations and severe quenchings the RT value of- $\sigma$ is proportional to $c_{2+}$ suggesting the activity of a Snoek-type reorientation of dipoles within the stress fields of dislocations. For larger concentration of dipoles the aggregation process occurs yielding either some changes of the $\sigma_{0}$ increments $\left(\mathrm{NaCl}: \mathrm{Sr}^{2+}\right)$ or leaving them unchanged with respect to the activity of isolated dipoles $\left(\mathrm{NaCl}: \mathrm{Eu}^{2+}\right)$. These differences in the behaviour of the first products of aggregation are probably related with a different strength of the mutual interactions between the dipoles. 

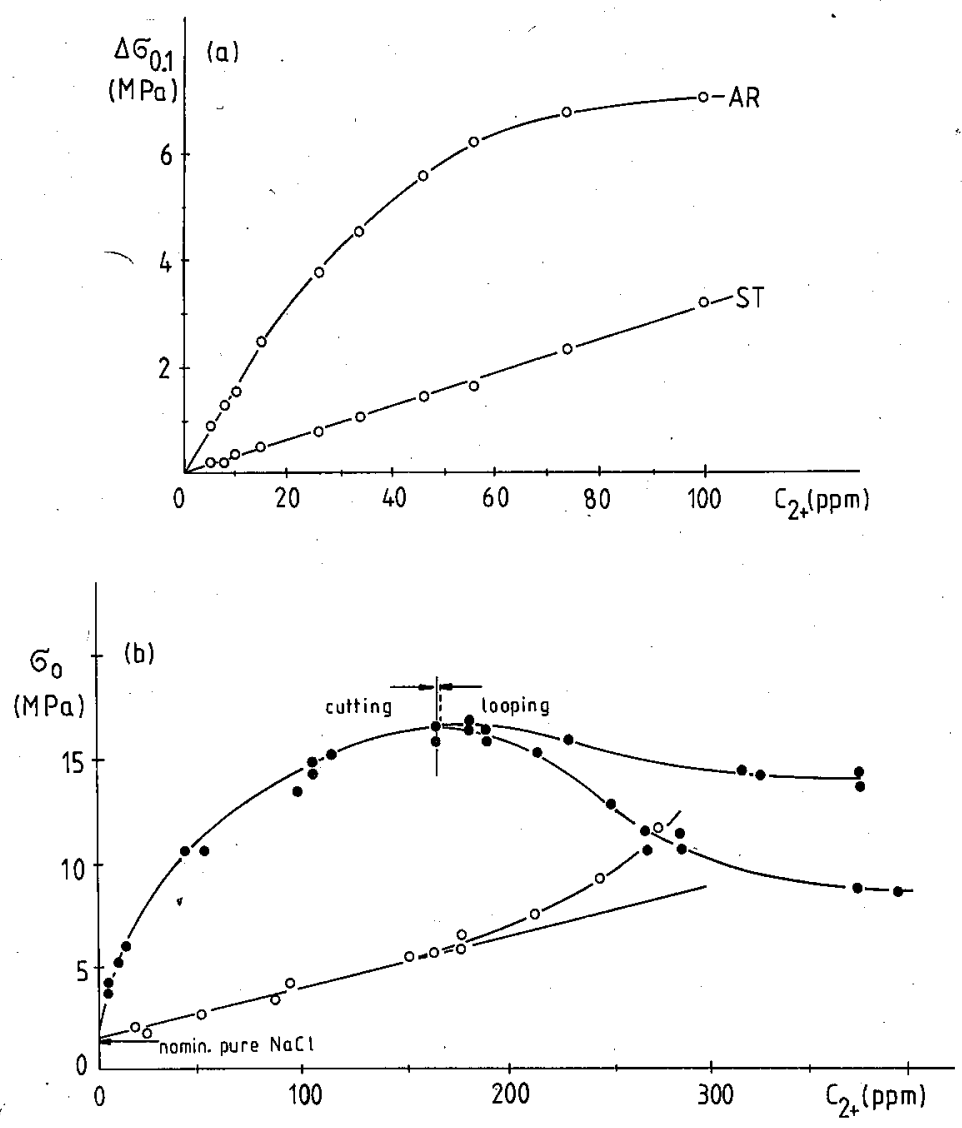

Fig. 1. Concentration dependences of the room temperature $0.1 \%$ proof stress of ARand ST-samples of $\mathrm{NaCl}: \mathrm{Sr}^{2+}$ (a) and $\mathrm{NaCl}: \mathrm{Eu}^{2+}$ (b) crystals; for (a) : $\Delta \sigma_{0}=\sigma_{0}$ (doped) - $\sigma_{0}$ (nomin. pure).

\subsubsection{AR-samples}

The quench-softening behaviour of the strontium-doped crystals suggests the activity of rather small particles which should be structurally coherent with the matrix. Taking $\sigma_{0} \propto 1 / z^{0.5}$, where $z$ is the number of dipoles per one particle, their size could be estimated from the following equation:

$$
z_{2}=\left(\sigma_{1} / \sigma_{2}\right)^{2} z_{1}
$$

where the subscripts 1 and 2 refer to ST- and AR-samples, respectively. Assuming that trimers $\left(z_{1}=3\right)$ are the stable aggregates in ST-samples, $z_{2}=4.7 \approx 5$, i.e. the pentamers should be characteristic for the AR-state.

On the other hand, the hardening-to-softening transition, typical of the europium-doped crystals, resembles the overaging behaviour of many $f c c$ metal alloys. 
Assuming that similarinteraction mechanisms are operative in the systems considered, the increasing and decreasing parts of the $\sigma_{0}\left(c_{2+}\right)$-plot (Fig. 1), should correspond to the cutting [4] and looping [5] of moving dislocations during their contact with the particles characteristic of slightly- and heavily-doped samples, respectively. These assignments correspond well with the X-ray diffraction- and optical absorption-data [9-11]. Namely, for AR-samples three phases have been identified, two of which are structurally coherent with the matrix (the $\mathrm{Na}_{2} \mathrm{EuCl}_{4}$ and $\mathrm{EuCl}_{2}$ of the $\mathrm{CaF}_{2}$-type) and one - incoherent (the orthorhombic $\mathrm{EuCl}_{2}$ ). From the point of view of the dimensions, all mentioned particles could be either coherent or incoherent with the matrix.

\subsection{Effect of $T_{\text {an }}$ for $\mathrm{NaCl}: \mathrm{Eu}^{2+}$}

The mechanical response of $\mathrm{NaCl}: \mathrm{Eu}^{2+}$ crystals, containing growing particles of the phases mentioned above, was studied for ST-samples subjected to different thermal pretreatment. Figure 2 shows some $\sigma_{0}\left(t_{\mathrm{an}}\right)$-plots for heavily-doped $\left(c_{2+} \geq 180 \mathrm{mppm}\right)$ samples annealed at various temperatures $T_{\mathrm{an}} ; t_{\mathrm{an}}$ denotes the annealing time.

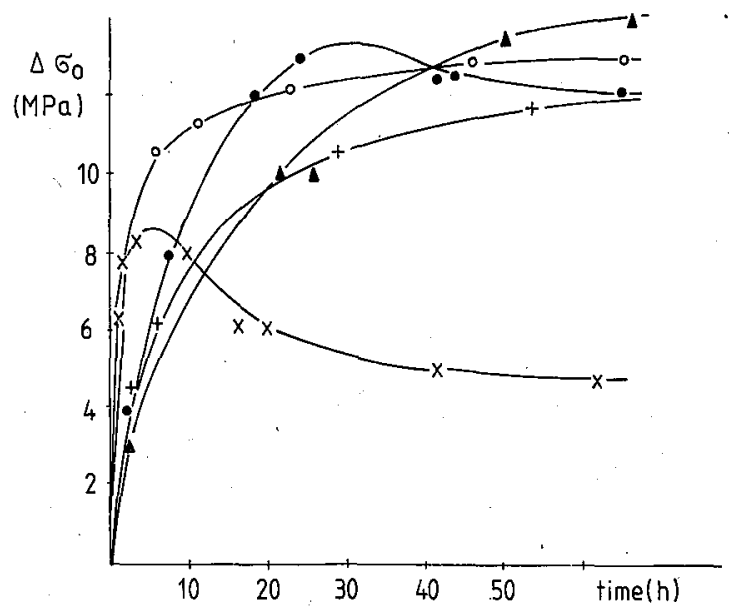

Fig. 2. $\Delta \sigma_{0}\left(t_{\mathrm{an}}\right)$-dependences of heavily-doped $\mathrm{NaCl}: \mathrm{Eu}^{2+}-\mathrm{ST}$-samples additionally annealed at $T_{\mathrm{an}}=323(+), 343(\Delta), 403(0), 473(\bullet)$ and $523(\times) \mathrm{K}$.

Taking into account these data as well as the ionic thermocurrent-kinetics, detected previously [6], the strengthening-to-softening transition could be understood in frames of the following precipitation sequence. Isolated I.V. dipoles and small aggregates (probably linear dimers) move towards dislocations, become stabilized there and transform into quadripoles (i.e. planar dimers) structurally equivalent to the $\mathrm{Na}_{2} \mathrm{EuCl}_{4}$ clusters. Growth of these particles is accompanied by the appearance of the extraordinary $\mathrm{EuCl}_{2}$ phase (with the structure of $\mathrm{CaF}_{2}$-type), and, finally, the orthorhombic $\mathrm{EuCl}_{2}$ particles. 


\subsection{Effect of $T_{\text {test }}$ for $\mathrm{NaCl}: \mathrm{Sr}^{2+}$}

Figure 3 shows the $\sigma_{0}\left(T_{\text {test }}\right)$-plots obtained for ST- and AR-samples of variously doped $\mathrm{NaCl}: \mathrm{Sr}^{2+}$ crystals. Careful analysis of the ST-data suggests the following interaction mechanisms might be ascribed to the various $T_{\text {test-ranges }}$ distinguished:

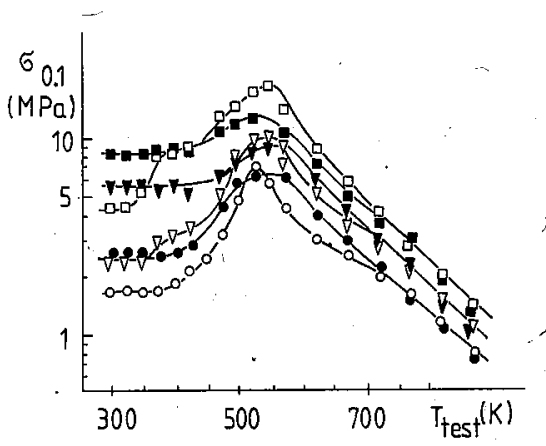

Fig. 3. $\sigma_{0}\left(T_{\text {test }}\right)$-dependences for AR- and ST-samples (full and open marks, respectively) of $\mathrm{NaCl}$ crystals containing 10 (@), 34 (@) and 100 (@) mole ppm of strontium.

- For the nearly temperature independent range the induced Snoek reorientation of dipoles in the stress field of dislocations determines the crystal strength [2].

- The maximum value of $\sigma_{0}$ and the related phenomena (serrated flow, for instance) agree well with the dynamic Cottrell-Bilby locking of dislocations [3].

- The high-temperature decrease of $\sigma_{0}$ corresponds to the dragging of the Cottrell atmospheres formed and to the thermal dissociation of the active dipoles.

It is clear that the pertinent interaction mechanism is determined by the temperature dependence of both the velocity of dislocations and the mobility of the impurity-related obstacles. Changes of these mechanisms occur at characteristic temperatures $\left(T_{\mathrm{s}-\mathrm{r}}\right.$ and $\left.T_{\mathrm{l}-\mathrm{r}}\right)$ at which the waiting time of dislocations in front of the dipoles equalizes the relaxation times of impurities for reorientation (short-range) and migration (long-range), respectively. The waiting time $t_{\mathrm{d}}$ of dislocations was calculated from the expression for the macroscopic strain rate $\left(\varepsilon=\phi b v \rho\right.$ with $\left.t_{\mathrm{d}}=1 / v\right)$ and the obstacle spacing $\left(1=b / c^{0.5}\right.$ for equidistant distribution). Numerical values obtained in this analysis are in good agreement with the experimental results; e.g. for $c_{2+}=10^{-4}$ and $\varepsilon=10^{-5} \mathrm{~s}^{-1}: t_{\mathrm{d}}=8 \times 10^{-3} \mathrm{~s}$ yielding $T_{\mathrm{s}-\mathrm{r}}=344 \mathrm{~K}$ and $T_{-\mathrm{r}}=543 \mathrm{~K}$. It should be noted that the expected Fleischer-type elastic interactions between nearly immobile obstacles (I.V. dipoles and small aggregates) and the moving dislocations are to be expected well below room temperature. 


\section{Summary}

The concentration dependences of $\sigma_{0}$ and the temperature-induced effects have substantiated the general conclusion drawn by W. Metag [12] that divalent impurities harden alkali halides intensively.

It has been evidenced that for ST-samples essentially a Snoek-like reorientation of dipoles defines the room temperature crystal strength. In contrary, for AR-samples differences in the mechanical response must be expected in different systems, and universal explanation of the strengthening effects will seldom be applicable. Moreover, one has to remember that the available theories are not completely rigorous and hence the models considered may not be realistic enough.

\section{Acknowledgments}

The authors wish to express their thanks to Mrs T. Morawska-Kowal for supplying the crystals.

\section{References}

[1] R.L. Fleischer, Acta Metall. 9, 996 (1961).

[2] G. Schoeck, A. Seeger, Acta Metall. 7, 469 (1959).

[3] A.H. Cottrell, B.A. Bilby, Proc. R. Soc. A 62, 49 (1949).

[4] A. Kelly, R.B. Nicholson, Strengthening Methods in Crystals, (Material Science Series) Elsevier, Amsterdam 1971.

[5] E. Orowan, Proc. of Symp. on Intermal Stresses in Metals and Alloys, Institute of Metals, London 1948, p. 451.

[6] A. Gubański, M. Suszyńska, D. Nowak-Wożny, Bull. Acad. Polon. Sci., Ser. Sci. Chim. 37, 393 (1989).

[7] M. Suszyńska, A. Gubański, H. Opyrchał, Cryst. Res. Technol. 22, 1229 (1987).

[8] R. Labusch, Phys. Status Solidi 41, 659 (1970).

[9] M. Suszyńska, H. Opyrchal, M. Czapelski, M. Manfredi, J. Mater. Sci. 22, 715 (1987).

[10] J. Stępień-Damm, K. Lukaszewicz, Acta Crystallogr. A 36, 54 (1980).

[11] J.M. Garcia, J.A. Hernandez, E.H. Carillo, J.O. Rubio, Phys. Rev. $B$ 21, 5012 (1980).

[12] W. Metag, Z. Phys. 78, 363 (1932) 